\title{
PRUEBAS DE BOMBEO EN POZOS DE GRAN DIÁMETRO
}

\author{
Gunther Schosinsky
}

\author{
Escuela Centoamericana de Geología \\ E-Mail: gschosin@geologia.ucr.ac.cr
}

\begin{abstract}
Well drilling increases highly the cost of an investigation. For this reason, existing wells are used for investigation in Central America, especially in the rural areas, where almost all wells are excavated and have a large diameter. Wells of large diameter store a considerable quantity of water in the casing, which affects the analysis of the pumping test with the existing conventional methods, which assumes no storage.

The existing method to analyze pumping tests in wells of large diameter requires long pumping periods, sometimes days, to obtain reliable results. These long duration tests are expensive and time-consuming. The methodology presented here permits the analysis of pumping tests of short duration to obtain accurate aquifer parameters.

The proposed methodology uses a model of wells of large diameter using cells of concentric rings. With this model families of curves were constructed that allow the analysis of pumping test in those wells where the storage in the casing affects the drawdown of the well. The proposed method used a new function, Stored Volume / Pumping Volume, that allows a good determination of the storage coefficient in wells of large diameter. When the value of Stored Volume / Pumping Volume is less than 0.05, the effect of the storage is practically imperceptible, and the pumping test can be analyzed by conventional methods, such as Theis or Jacob. The proposed method allows the analysis of pumping tests of short duration in wells of large diameter, with the advantage that the wells of different storativity or storage coefficient have a unique curve matching. When the values of Stored Volume / Pumping Volume are greater than 0.05 , the propose method allows the determination of the storage coefficient even in wells of smaller diameter $(15-25 \mathrm{~cm})$, in cases when conventional methods would usually not function.
\end{abstract}

RESUMEN: En Centro América, especialmente en la zona rural, la gran mayoría de los pozos son excavados. Estos pozos, al ser de gran diámetro, almacenan una considerable cantidad de agua dentro del pozo, afectando el análisis de la prueba de bombeo.

Existe un método para analizar pruebas de bombeo en pozos de gran diámetro que requiere un bombeo continuo durante largo tiempo, algunas veces hasta días, lo que es improducente en una campaña de recolección de datos. Aquí se presenta una metodología que permite analizar con éxito una prueba de bombeo de corta duración en pozos de gran diámetro.

Se desarrolló un modelo de pozos de gran diámetro, utilizando celdas de anillos concéntricos. Reproduciendo pruebas de bombeo, se obtuvieron funciones con las que se construyeron curvas patrón que permiten un análisis de mayor precisión de las pruebas de bombeo, donde el almacenamiento en el ademe de los pozos influye en los abatimientos del pozo. El método propuesto utiliza una nueva función, Volumen almacenado / Volumen bombeado, que permite determinar con mayor precisión el coeficiente de almacenamiento. Cuando el valor de Volumen almacenado / Volumen bombeado, es menor de 0,05, el efecto de almacenamiento prácticamente ha desaparecido, por lo que la prueba de bombeo puede ser analizada por los métodos convencionales de Theis o Jacob. Con valores de Volumen almacenado / Volumen bombeado mayores de 0,05, este método permite determinar el coeficiente de almacenamiento en los pozos de bombeo, aún en diámetros de perforación generalmente utilizados $(15 \mathrm{~cm}-25 \mathrm{~cm})$ que con los métodos convencionales no permiten la determinación del coeficiente de almacenamiento en forma precisa para estos diámetros. 


\section{INTRODUCCIÓN}

En el estudio de las aguas subterráneas es indispensable la información de parámetros hidrogeológicos, tales como el nivel estático, transmisibilidad y coeficiente de almacenamiento. Con estos se predice el comportamiento de un acuífero, en relación con su producción sostenible. Estos parámetros se obtienen mediante pruebas de bombeo realizadas en pozos.

En un proyecto de evaluación del recurso hidrogeológico, se requieren varias pruebas de bombeo en diferentes sitios del área de estudio. Por ser la perforación de pozos para investigación sumamente costosa, se utilizan los pozos existentes en la zona. Sin embargo, en Centro América, especialmente en la zona rural, la gran mayoría de los pozos son excavados. Estos pozos, al ser de gran diámetro, almacenan una considerable cantidad de agua dentro del pozo, afectando el análisis de la prueba de bombeo, por asumir la metodología convencional que el pozo no almacena agua dentro de él.

Existe un método para analizar pruebas de bombeo en pozos de gran diámetro PapadopulosCooper (Kruseman \& De Rider, 1994), que para aplicarlo con éxito, requiere un bombeo continuo durante largo tiempo, algunas veces hasta días, lo que es improducente en una campaña de recolección de datos.

Este estudio, tiene la finalidad de desarrollar una metodología, que permita analizar una prueba de bombeo de corta duración (de una a dos horas) en pozos de gran diámetro, para obtener los parámetros adecuados del acuífero. Con lo cual se disminuye el tiempo y costo de la recolección de datos.

Se esarrolló un modelo de pozos de gran diámetro, utilizando celdas de anillos concéntricos, en que se pudiese variar el espesor de cada uno de ellos, llegando a espesores de $5 \mathrm{~cm}$ en los anillos cercanos al pozo. Reproduciendo pruebas de bombeo con el modelo, se obtuvieron funciones con las que se construyeron curvas patrón que permiten un análisis de mayor precisión de las pruebas de bombeo, donde el almacenamiento en el ademe de los pozos, influye en los abatimientos del pozo.

\section{ANTECEDENTES}

Uno de los métodos más utilizados para analizar pruebas de bombeo en pozos de gran diámetro es el de Papadopulos-Cooper (Kruseman \& De Ridder, 1994). En esta metodología se realiza una prueba de bombeo a caudal constante en un pozo de gran diámetro, llevando un registro del tiempo desde el inicio de bombeo y cada uno de los abatimientos ocurridos en cada tiempo.

\section{Método de Papadopulos}

El método de Papadopulos consiste en graficar en papel doblemente logarítmico, el abatimiento observado durante la prueba de bombeo en el eje vertical, contra el tiempo en que ocurre cada abatimiento, en el eje horizontal. La escala a utilizar ha de ser la misma en que se encuentran graficadas la familia de curvas patrón de Papadopulos (Fig 1). Posteriormente, se sobrepone la gráfica de los abatimientos obtenidos de la prueba de bombeo, sobre las curvas patrón, haciendo coincidir la curva de los abatimientos con alguna de ellas.

Conociendo la curva de la Familia Papadopulos que sigue el mismo patrón de los abatimientos obtenidos en la prueba de bombeo y aplicando una fórmula sencilla, se determinan los parámetros hidrogeológicos de transmisibilidad y coeficiente de almacenamiento (Kruseman \& De Ridder, 1994).

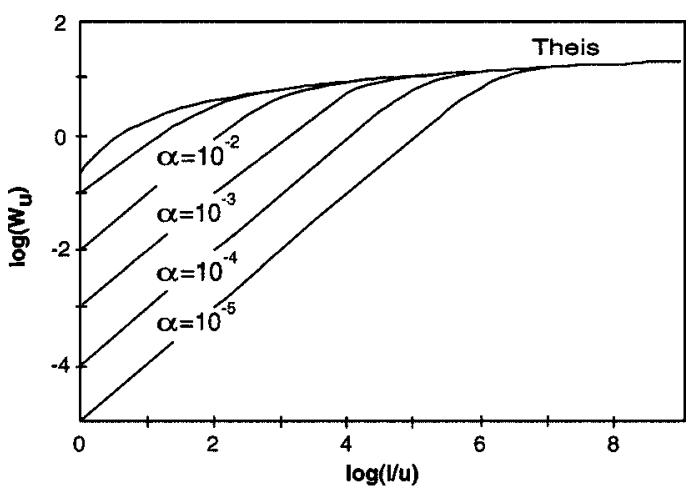

Fig. 1: Familia de curvas patrón de Papadopulos. Valores tomados del Anexo 11.1 de Kruseman \& De Ridder (1994). El valor de $1 / \mathrm{u}$ corresponde al inverso de la función $\mathrm{u}=\mathrm{r}_{\mathrm{w}}^{2} \mathrm{~S} /(4 \mathrm{Tt})$. 
En pruebas de bombeo cortas, es muy difícil hacer coincidir los abatimientos con un solo patrón de la curva de la Familia Papadopulos, debido a que ellas difieren levemente en su forma (Fig. 1). Moviéndose de una curva patrón a otra, los resultados del coeficiente de almacenamiento pueden variar en más de un orden de magnitud, lo que hace dudoso la selección del coeficiente de almacenamiento en pruebas cortas de bombeo. En el caso de la transmisibilidad, esta es menos sensible a la selección de la curva patrón (Kruseman \& De Ridder, 1994).

En la figura 1 las diferentes curvas tienen igual conformación en tiempos de corta duración, los abatimientos obtenidos en una prueba de bombeo de corta duración pueden sobreponerse en cualquiera de ellas.

La ecuación de Papadopulos es para determinar el abatimiento en un pozo con almacenamiento de agua dentro del agujero (Kruseman \& De Ridder, 1994); esta dada por:

$$
\begin{gathered}
s=\frac{Q}{4 \pi T} F(\alpha, u) \\
u=\frac{r_{w}^{2} S}{4 T t}
\end{gathered}
$$

$\mathrm{s}[\mathrm{m}]=\quad$ Abatimiento en el pozo de bombeo. $\mathrm{Q}\left[\mathrm{m}^{3} /\right.$ día $]=$ Caudal constante de bombeo.

$\mathrm{T}\left[\mathrm{m}^{2} /\right.$ día $]=$ Transmisibilidad del acuífero.

$\mathrm{a}[\mathrm{m} / \mathrm{m}]=\quad \mathrm{S} \mathrm{r}^{2}{ }_{\mathrm{w}} / \mathrm{r}^{2}{ }_{\mathrm{c}}$

$r_{w}[\mathrm{~m}]=\quad$ Radio efectivo de la rejilla (o parte abierta en el acuífero captado) del pozo

$r_{c}[\mathrm{~m}]=\quad$ Radio efectivo del almacenamiento (ademe) en donde se encuentra el nivel de agua.

$\mathrm{S}=\quad$ Coeficiente de almacenamiento

$\mathrm{t}$ [días $=$ Tiempo de bombeo en días

Los supuestos de la ecuación de Papadopulos-Cooper (Kruseman \& De Ridder, 1994) son los siguientes:

1) El acuífero es de extensión infinita.

2) El acuífero es homogéneo, isotrópico y de transmisibilidad uniforme sobre el área influenciada por el bombeo.
3) Antes del bombeo la superficie piezométrica o freática es horizontal o casi horizontal.

4) El bombeo en el pozo se realiza a un caudal constante.

5) El pozo es completamente penetrante en el acuífero.

6) No existe recarga en el acuífero en la zona influenciada por el bombeo.

7) Las pérdidas por resistencia de la entrada del agua al pozo son despreciables.

8) El diámetro del pozo no se puede considerar infinitesimal, por lo que no se puede despreciar el agua almacenada dentro del agujero del pozo.

9) El flujo del acuífero hacia el pozo es transiente.

\section{METODOLOGÍA PROPUESTA}

En la ecuación (1) se puede observar que el abatimiento en un pozo de gran diámetro depende de la función $\mathrm{F}(\mathrm{a}, \mathrm{u})$ la cual depende de la función $\mathrm{u}=\mathrm{r}_{\mathrm{w}}^{2} \mathrm{~S} /(4 \mathrm{Tt}) \mathrm{y}$ del coeficiente $\mathrm{a}=\mathrm{S}$ $\mathrm{r}_{\mathrm{w}}^{2} / \mathrm{r}^{2}{ }_{\mathrm{c}}$. Si el radio efectivo de la rejilla $\left(\mathrm{r}_{\mathrm{w}}\right)$ es igual al radio efectivo del almacenamiento $\left(r_{c}\right)$ el coeficiente a, igualaría al coeficiente de almacenamiento $(\mathrm{S})$, dependiendo entonces la función de pozo $\mathrm{F}(\mathrm{a}, \mathrm{u})$ de $\mathrm{u}$ y del coeficiente de almacenamiento.

De la ecuación (1) tenemos que:

$$
F(\alpha, u)=\frac{4 \pi T s}{Q}
$$

Multiplicando ambos términos de la ecuación por la función u tenemos la ecuación 2:

$$
F(\alpha, u) u=\frac{s \pi r_{w}^{2} S}{Q t}
$$

Donde:

$$
\frac{F(\alpha, u) u}{S}=\frac{s \pi r_{w}^{2}}{Q t}=
$$

Volumen almacenado / Volumen bombeado (3)

Los valores del volumen de agua almacenado y el volumen bombeado se pueden obtener 
directamente del campo durante la prueba de bombeo

La función $\mathrm{F}(\alpha, \mathrm{u}) \mathrm{u} / \mathrm{S}$ fue obtenida mediante un modelo computarizado construido con base en celdas formadas por anillos concéntricos. Con los datos obtenidos mediante el modelo se construyeron curvas para diferentes valores de a.

\section{Modelo computarizado}

Se construyó un modelo computarizado de pozos de gran diámetro utilizando hojas electrónicas del programa EXCEL, donde la primera celda está formada por un círculo que representa al pozo. Las otras celdas están formadas de anillos concéntricos de espesor variable, ajustable al valor deseado; generalmente se utilizaron los anillos cercanos al pozo con espesor de $10 \mathrm{~cm}$. El modelo se basa en las ecuaciones de Darcy, realizando balances de agua entre celda y celda a incrementos de tiempo muy cortos (dt), generalmente menores de un segundo.

Las ecuaciones utilizadas en las celdas son las siguientes:

$$
\begin{gathered}
h_{p t}=h_{p}+\frac{2 T d t}{r} \cdot \frac{\left(h_{1}-h_{p}\right)}{\left(d_{1}-r\right)}-\frac{Q d t}{\pi r^{2}} \quad(4) \\
h_{1 t}=h_{1}+\frac{2 T d t}{\left(R E_{1}^{2}-r^{2}\right) S}\left[\frac{R E_{1}\left(h_{2}-h_{1}\right)}{\left(d_{2}-d_{1}\right)}-\frac{r\left(h_{1}-h_{p}\right)}{\left(d_{1}-r\right)}\right] \quad(5) \\
h_{i t}=h_{i}+\frac{2 T d t}{\left(R E_{i}^{2}-R I_{i}^{2}\right) S} \cdot\left[\frac{R E_{i}\left(h_{i+1}-h_{i}\right)}{\left(d_{i+1}\right)-d_{i}}-\frac{R I_{i}\left(h_{i}-h_{i-1}\right)}{\left(d_{1}-d_{i-1}\right)}\right](6)
\end{gathered}
$$

(4) Ecuación a utilizar en la celda que representa el pozo

(5) Ecuación a utilizar en la celda que representa el anillo dentro de la formación acuífera (celda 1) que rodea al pozo, generalmente a este anillo se le asignan espesores de $10 \mathrm{~cm}$ o menos.

(6) Ecuación a utilizar en las celdas siguientes a la celda 1, ubicadas en el acuífero y formadas por anillos concéntricos alrededor del pozo (celdas i ). $\mathrm{h}_{\mathrm{p}}[\mathrm{m}]=\quad$ Elevación del nivel de agua dentro del pozo antes de un tiempo dt.

$\mathrm{h}_{\mathrm{pt}}[\mathrm{m}]=\quad$ Elevación del nivel de agua dentro del pozo después de un tiempo dt.

$\mathrm{h}_{\mathrm{i}}[\mathrm{m}]=\quad$ Elevación del nivel de agua en la celda i antes de un tiempo dt.

$\mathrm{h}_{\mathrm{it}}[\mathrm{m}]=\quad$ Elevación del nivel de agua en la celda i después de un tiempo dt.

$\mathrm{r}[\mathrm{m}]=\quad$ Radio efectivo del pozo de gran diámetro.

$\mathrm{dt}[\mathrm{dí} a \mathrm{~s}]=$ Incremento de tiempo en cada iteración, generalmente menor de1 segundo.

$\mathrm{T}\left[\mathrm{m}^{2} / \mathrm{d}\right]=$ Transmisibilidad

$\mathrm{RE}_{\mathrm{i}}[\mathrm{m}]=$ Radio exterior del anillo que conforma la celda $i$.

$\mathrm{RI}_{\mathrm{i}}[\mathrm{m}]=\quad$ Radio interior del anillo que conforma la celda $i$.

$\mathrm{d}_{\mathrm{i}}[\mathrm{m}]=\quad$ Distancia del centro del pozo al centro del anillo que conforma la celda $\mathrm{i}$.

El modelo utiliza las siguientes suposiciones:

1) El acuífero es de extensión infinita.

2) El acuífero es homogéneo, isotrópico y de transmisibilidad uniforme sobre el área influenciada por el bombeo.

3) Antes del bombeo la superficie piezométrica o freática es horizontal o casi horizontal.

4) El bombeo en el pozo se realiza a un caudal constante.

5) El pozo es completamente penetrante en el acuífero.

6) No existe recarga en el acuífero en la zona influenciada por el bombeo.

7) Las pérdidas por resistencia de la entrada del agua al pozo son despreciables.

8) El pozo de bombeo no tiene limitación de diámetro.

9) Los abatimientos se miden en el pozo de bombeo.

De esta forma se reprodujeron pruebas de bombeo con diferentes diámetros de pozo, valores de transmisibilidad, coeficientes de almacenamiento y caudal de bombeo. Los valores de abatimiento obtenidos con el modelo, 
coinciden en todos los casos con las curvas de Papadopulos (Fig 1), para pruebas de bombeo en pozos de gran diámetro (Kruseman \& De Ridder, 1994).

Para ilustrar la confiabilidad del modelo, se muestra en la figura 2 los valores de abatimiento (mostrado con cuadros), los que fueron obtenidos en una simulación realizada, comparándolos con la curva de Theis (línea gruesa) y Papadopulos (línea fina). Los valores de abatimiento simulados coinciden perfectamente con los valores de la curva patrón, a = 0,1, de la familia de Papadopulos (Fig 1 y Fig 2). En la figura 2, se muestra el abatimiento obtenido en el modelo, utilizando los siguientes valores de parámetros hidrogeológicos: transmisibilidad 20 $\mathrm{m}^{2} /$ día, coeficiente de almacenamiento 0,1 , caudal $0,461 / \mathrm{s}$ y el radio del pozo $0,70 \mathrm{~m}$. El tiempo simulado de la prueba de bombeo es de 120 minutos. Se puede observar que los abatimientos se sobreponen perfectamente a la curva de Papadopulos a = 0,1. Además, los abatimientos inician el ajuste a la curva de Theis, aproximadamente a los 600 minutos, lo que requiere una prueba de bombeo de 1200 minutos para poderla analizar correctamente con el método de Papadopulos.

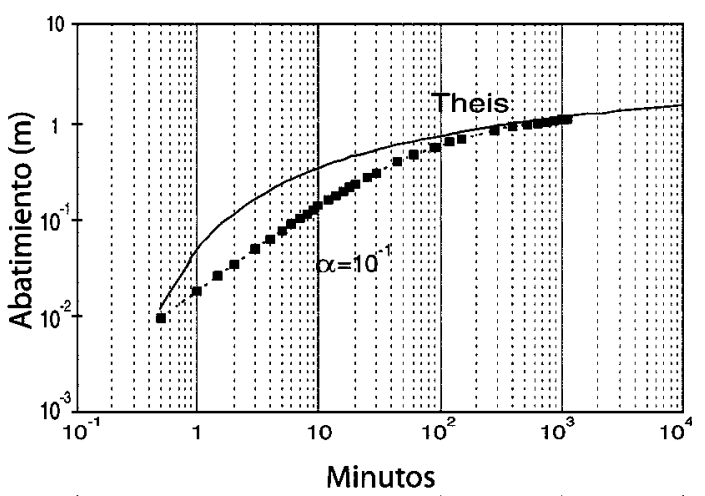

Fig. 2: Abatimientos simulados con el modelo, utilizando los parámetros hidrogeológicos indicados en el recuadro de la figura. Obsérvese la sobreposición del abatimiento sobre la curva de Theis y Papadopulos. Los valores de T y S obtenidos con la sobreposición son los mismos que los utilizados en la simulación.

Con el modelo se obtiene la función $\mathrm{F}(\mathrm{a}$, $\mathrm{u}) \mathrm{u} / \mathrm{S}$ y la función $\mathrm{F}(\alpha, \mathrm{u})$, valores que se encuentran en el cuadro1. Cabe aclarar que para cada valor de u y a existe un único valor de $\mathrm{F}(\alpha, \mathrm{u})$. Además, para cada valor de $\mathrm{F}(\alpha, \mathrm{u})$ y $\mathrm{S}$, existe un único valor de $\mathrm{F}(\alpha, \mathrm{u}) \mathrm{u} / \mathrm{S}$.

Los valores obtenidos del modelo, permiten la construcción de curvas patrón para analizar pruebas de bombeo con almacenamiento de agua en el pozo.

Cuadro 1

Valores de la función Volumen almacenado / Volumen bombeado vs. F(u,a), para diferentes valores de $\mathrm{S}$

\begin{tabular}{lcccccccc}
\hline & $\mathrm{S}=10-5$ & $\mathrm{~S}=10-4$ & $\mathrm{~S}=10-3$ & $\mathrm{~S}=10-2$ & $\mathrm{~S}=0,05$ & $\mathrm{~S}=0,1$ & $\mathrm{~S}=0,2$ & $\mathrm{~S}=0,3$ \\
\hline Valm / Vbom & $\mathrm{F}(\mathrm{u}, \alpha)$ & $\mathrm{F}(\mathrm{u}, \alpha)$ & $\mathrm{F}(\mathrm{u}, \alpha)$ & $\mathrm{F}(\mathrm{u}, \alpha)$ & $\mathrm{F}(\mathrm{u}, \alpha)$ & $\mathrm{F}(\mathrm{u}, \alpha)$ & $\mathrm{F}(\mathrm{u}, \alpha)$ & $\mathrm{F}(\mathrm{u}, \alpha)$ \\
& & & & & & & & \\
$9,9 * 10-1$ & 0,154 & 0,103 & 0,053 & 0,025 & 0,021 & 0,020 & 0,019 & 0,019 \\
$9,8 * 10-1$ & 0,343 & 0,240 & 0,137 & 0,063 & 0,043 & 0,042 & 0,040 & 0,040 \\
$9,7 * 10-1$ & 0,543 & 0,389 & 0,232 & 0,108 & 0,070 & 0,065 & 0,062 & 0,062 \\
$9,5 * 10-1$ & 0,963 & 0,705 & 0,444 & 0,217 & 0,131 & 0,115 & 0,107 & 0,104 \\
$9,3 * 10-1$ & 1,396 & 1,037 & 0,673 & 0,343 & 0,199 & 0,171 & 0,154 & 0,149 \\
$9,2 * 10-1$ & 1,615 & 1,208 & 0,793 & 0,410 & 0,237 & 0,200 & 0,178 \\
$9,1 * 10-1$ & 1,837 & 1,380 & 0,916 & 0,481 & 0,276 & 0,230 & 0,204 \\
$9,0 * 10-1$ & 2,059 & 1,555 & 1,040 & 0,554 & 0,316 & 0,262 & 0,229 & 0,171 \\
$8,7 * 10-1$ & 2,732 & 2,085 & 1,425 & 0,787 & 0,449 & 0,363 & 0,310 & 0,218 \\
$8,5 * 10-1$ & 3,182 & 2,443 & 1,688 & 0,952 & 0,546 & 0,436 & 0,366 \\
$8,3 * 10-1$ & 3,632 & 2,803 & 1,956 & 1,122 & 0,648 & 0,513 & 0,426 & 0,340 \\
$8,2 * 10-1$ & 3,857 & 2,984 & 2,090 & 1,209 & 0,701 & 0,553 & 0,456 & 0,393 \\
$8,1 * 10-1$ & 4,082 & 3,164 & 2,226 & 1,297 & 0,755 & 0,595 & 0,487 & 0,446 \\
\hline
\end{tabular}




\begin{tabular}{|c|c|c|c|c|c|c|c|c|}
\hline & $S=10-5$ & $S=10-4$ & $S=10-3$ & $S=10-2$ & $\mathrm{~S}=0,05$ & $\mathrm{~S}=0,1$ & $\mathrm{~S}=0,2$ & $\mathrm{~S}=0,3$ \\
\hline Valm / Vbom & $\mathrm{F}(\mathrm{u}, \alpha)$ & $\mathrm{F}(\mathrm{u}, \alpha)$ & $\mathrm{F}(\mathrm{u}, \alpha)$ & $\mathrm{F}(\mathrm{u}, \alpha)$ & $\mathrm{F}(\mathrm{u}, \alpha)$ & $\mathrm{F}(\mathrm{u}, \alpha)$ & $\mathrm{F}(\mathrm{u}, \alpha)$ & $\mathrm{F}(\mathrm{u}, \alpha)$ \\
\hline $8,0 * 10-1$ & 4,307 & 3,345 & 2,362 & 1,386 & 0,810 & 0,637 & 0,519 & 0,474 \\
\hline $7,7 * 10-1$ & 4,977 & 3,887 & 2,772 & 1,659 & 0,983 & 0,769 & 0,619 & 0,559 \\
\hline $7,5 * 10-1$ & 5,421 & 4,247 & 3,047 & 1,845 & 1,104 & 0,862 & 0,688 & 0,618 \\
\hline $7,3 * 10-1$ & 5,861 & 4,606 & 3,323 & 2,034 & 1,228 & 0,958 & 0,761 & 0,679 \\
\hline $7,2 * 10-1$ & 6,080 & 4,785 & 3,462 & 2,130 & 1,291 & 1,008 & 0,798 & 0,711 \\
\hline $7,1 * 10-1$ & 6,298 & 4,964 & 3,600 & 2,226 & 1,356 & 1,058 & 0,836 & 0,743 \\
\hline $7,0 * 10-1$ & 6,515 & 5,142 & 3,738 & 2,326 & 1,423 & 1,111 & 0,874 & 0,773 \\
\hline $6,7 * 10-1$ & 7,159 & 5,672 & 4,152 & 2,616 & 1,621 & 1,268 & 0,994 & 0,875 \\
\hline $6,5 * 10-1$ & 7,582 & 6,022 & 4,428 & 2,811 & 1,757 & 1,377 & 1,077 & 0,945 \\
\hline $6,3 * 10-1$ & 8,000 & 6,368 & 4,702 & 3,007 & 1,895 & 1,489 & 1,163 & 1,018 \\
\hline $6,2 * 10-1$ & 8,206 & 6,540 & 4,839 & 3,106 & 1,966 & 1,546 & 1,207 & 1,055 \\
\hline $6,1 * 10-1$ & 8,411 & 6,711 & 4,975 & 3,205 & 2,037 & 1,604 & 1,252 & 1,093 \\
\hline $6,0 * 10-1$ & 8,615 & 6,881 & 5,111 & 3,310 & 2,112 & 1,665 & 1,298 & 1,130 \\
\hline $5,7 * 10-1$ & 9,216 & 7,385 & 5,515 & 3,612 & 2,332 & 1,846 & 1,438 & 1,248 \\
\hline $5,5 * 10-1$ & 9,608 & 7,715 & 5,783 & 3,813 & 2,482 & 1,971 & 1,536 & 1,330 \\
\hline $5,3 * 10-1$ & 9,993 & 8,040 & 6,047 & 4,015 & 2,634 & 2,098 & 1,636 & 1,415 \\
\hline $5,2 * 10-1$ & 10,182 & 8,201 & 6,179 & 4,116 & 2,710 & 2,162 & 1,687 & 1,459 \\
\hline $5,1 * 10-1$ & 10,370 & 8,360 & 6,310 & 4,216 & 2,788 & 2,227 & 1,740 & 1,503 \\
\hline $5,0 * 10-1$ & 10,555 & 8,518 & 6,440 & 4,317 & 2,865 & 2,293 & 1,793 & 1,548 \\
\hline $4,7 * 10-1$ & 11,099 & 8,983 & 6,825 & 4,620 & 3,101 & 2,494 & 1,956 & 1,688 \\
\hline $4,5 * 10-1$ & 11,450 & 9,286 & 7,078 & 4,821 & 3,261 & 2,632 & 2,069 & 1,785 \\
\hline $4,3 * 10-1$ & 11,792 & 9,581 & 7,328 & 5,022 & 3,422 & 2,773 & 2,186 & 1,886 \\
\hline $4,2 * 10-1$ & 11,960 & 9,727 & 7,451 & 5,123 & 3,504 & 2,844 & 2,246 & 1,938 \\
\hline $4,1 * 10-1$ & 12,125 & 9,871 & 7,574 & 5,223 & 3,586 & 2,916 & 2,306 & 1,990 \\
\hline $4,0 * 10-1$ & 12,287 & 10,013 & 7,695 & 5,323 & 3,668 & 2,989 & 2,367 & 2,044 \\
\hline $3,7 * 10-1$ & 12,760 & 10,429 & 8,055 & 5,623 & 3,918 & 3,212 & 2,557 & 2,212 \\
\hline $3,5 * 10-1$ & 13,062 & 10,697 & 8,289 & 5,823 & 4,087 & 3,365 & 2,689 & 2,329 \\
\hline $3,3 * 10-1$ & 13,354 & 10,959 & 8,520 & 6,022 & 4,258 & 3,521 & 2,825 & 2,452 \\
\hline $3,2 * 10-1$ & 13,496 & 11,087 & 8,634 & 6,121 & 4,345 & 3,600 & 2,894 & 2,515 \\
\hline $3,1 * 10-1$ & 13,635 & 11,213 & 8,747 & 6,220 & 4,432 & 3,681 & 2,965 & 2,579 \\
\hline $3,0 * 10-1$ & 13,772 & 11,338 & 8,859 & 6,304 & 4,505 & 3,746 & 3,023 & 2,628 \\
\hline $2,7 * 10-1$ & 14,167 & 11,701 & 9,191 & 6,607 & 4,779 & 4,002 & 3,254 & 2,842 \\
\hline $2,5 * 10-1$ & 14,418 & 11,935 & 9,408 & 6,813 & 4,969 & 4,182 & 3,420 & 2,996 \\
\hline $2,3 * 10-1$ & 14,661 & 12,164 & 9,623 & 7,022 & 5,165 & 4,369 & 3,594 & 3,160 \\
\hline $2,2 * 10-1$ & 14,780 & 12,277 & 9,730 & 7,128 & 5,266 & 4,466 & 3,684 & 3,245 \\
\hline $2,1 * 10-1$ & 14,897 & 12,388 & 9,837 & 7,235 & 5,367 & 4,564 & 3,777 & 3,333 \\
\hline $2,0 * 10-1$ & 15,013 & 12,500 & 9,944 & 7,326 & 5,456 & 4,646 & 3,853 & 3,403 \\
\hline $1,7 * 10-1$ & 15,355 & 12,833 & 10,271 & 7,647 & 5,768 & 4,951 & 4,145 & 3,682 \\
\hline $1,5 * 10-1$ & 15,584 & 13,060 & 10,497 & 7,873 & 5,990 & 5,172 & 4,358 & 3,889 \\
\hline $1,3 * 10-1$ & 15,820 & 13,297 & 10,736 & 8,115 & 6,231 & 5,412 & 4,590 & 4,118 \\
\hline $1,2 * 10-1$ & 15,944 & 13,422 & 10,863 & 8,243 & 6,360 & 5,541 & 4,716 & 4,242 \\
\hline $1,1 * 10-1$ & 16,073 & 13,553 & 10,995 & 8,377 & 6,496 & 5,676 & 4,850 & 4,374 \\
\hline $1,0 * 10-1$ & 16,210 & 13,692 & 11,135 & 8,519 & 6,642 & 5,821 & 4,994 & 4,515 \\
\hline $7,0 * 10-2$ & 16,690 & 14,175 & 11,622 & 9,017 & 7,153 & 6,332 & 5,510 & 5,024 \\
\hline $5,0 * 10-2$ & 17,108 & 14,595 & 12,051 & 9,459 & 7,600 & 6,789 & 5,965 & 5,483 \\
\hline $3,0 * 10-2$ & 17,697 & 15,206 & 12,672 & 10,091 & 8,249 & 7,440 & 6,624 & 6,142 \\
\hline $2,0 * 10-2$ & 18,153 & 15,673 & 13,142 & 10,571 & 8,740 & 7,937 & 7,130 & 6,649 \\
\hline $1,0 * 10-2$ & 18,933 & 16,449 & 13,933 & 11,378 & 9,559 & 8,764 & 7,962 & 7,489 \\
\hline $7,0 * 10-3$ & 19,335 & 16,849 & 14,331 & 11,780 & 9,972 & 9,179 & 8,385 & 7,912 \\
\hline $5,0 * 10-3$ & 19,713 & 17,227 & 14,702 & 12,160 & 10,355 & 9,571 & 8,776 & 8,310 \\
\hline $3,0 * 10-3$ & 20,288 & 17,802 & 15,268 & 12,735 & 10,937 & 10,155 & 9,365 & 8,901 \\
\hline $2,0 * 10-3$ & 20,745 & 18,259 & 15,713 & 13,183 & 11,396 & 10,613 & 9,833 & 9,368 \\
\hline
\end{tabular}




\begin{tabular}{lcccccccc}
\hline & $\mathrm{S}=10-5$ & $\mathrm{~S}=10-4$ & $\mathrm{~S}=10-3$ & $\mathrm{~S}=10-2$ & $\mathrm{~S}=0,05$ & $\mathrm{~S}=0,1$ & $\mathrm{~S}=0,2$ & $\mathrm{~S}=0,3$ \\
Valm / Vbom & $\mathrm{F}(\mathrm{u}, \alpha)$ & $\mathrm{F}(\mathrm{u}, \alpha)$ & $\mathrm{F}(\mathrm{u}, \alpha)$ & $\mathrm{F}(\mathrm{u}, \alpha)$ & $\mathrm{F}(\mathrm{u}, \alpha)$ & $\mathrm{F}(\mathrm{u}, \alpha)$ & $\mathrm{F}(\mathrm{u}, \alpha)$ & $\mathrm{F}(\mathrm{u}, \alpha)$ \\
\hline $1,0 * 10-3$ & 21,525 & 19,039 & 16,503 & 13,953 & 12,169 & 11,398 & 10,616 & 10,159 \\
$7,0 * 10-4$ & 21,926 & 19,440 & 16,903 & 14,345 & 12,569 & 11,794 & 11,021 & 10,561 \\
$5,0 * 10-4$ & 22,305 & 19,819 & 17,281 & 14,711 & 12,941 & 12,170 & 11,399 & 10,943 \\
$3,0 * 10-4$ & 22,880 & 20,394 & 17,854 & 15,274 & 13,504 & 12,741 & 11,967 & 11,517 \\
$2,0 * 10-4$ & 23,336 & 20,850 & 18,309 & 15,717 & 13,955 & 13,187 & 12,421 & 11,967 \\
$1,0 * 10-4$ & 24,116 & 21,630 & 19,087 & 16,477 & 14,723 & 13,955 & 13,187 & 12,742 \\
$7,0 * 10-5$ & 24,518 & 22,032 & 19,487 & 16,868 & 15,118 & 14,346 & 13,581 & 13,134 \\
$5,0 * 10-5$ & 24,897 & 22,411 & 19,865 & 17,237 & 15,491 & 14,712 & 13,955 & 13,505 \\
$3,0 * 10-5$ & 25,472 & 22,985 & 20,438 & 17,797 & 16,057 & 15,275 & 14,514 & 14,071 \\
$2,0 * 10-5$ & 25,928 & 23,442 & 20,893 & 18,242 & 16,506 & 15,717 & 14,958 & 14,514 \\
$1,0 * 10-5$ & 26,708 & 24,222 & 21,671 & 19,002 & 17,274 & 16,477 & 15,719 & 15,280 \\
\hline
\end{tabular}

\section{CURVAS PATRÓN PARA ANÁLISIS DE PRUEBAS DE BOMBEO CON ALMACENAMIENTO DE AGUA EN EL POZO}

Las curvas patrón (Fig. 3) se construyeron graficando los valores de $\mathrm{F}(\alpha, \mathrm{u}) / \mathrm{S}$ en escala aritmética en el eje de las ordenadas y los valores de $\mathrm{F}(\alpha, \mathrm{u})$, en escala logarítmica, en el eje de las abscisas.

De acuerdo a la ecuación (3) tenemos:

$\mathrm{F}(\alpha, \mathrm{u}) / \mathrm{S}=$ Volumen almacenado / Volumen bombeado

Volumen almacenado $/$ Volumen bombeado $=$

$$
\frac{s \pi r_{w}^{2}}{Q t} \quad(7) \quad \alpha=\frac{S r_{w}^{2}}{r_{c}^{2}}
$$

$\mathrm{s}[\mathrm{m}]=\quad$ Abatimiento en el pozo de bombeo a un tiempo $t$. $S=\operatorname{ar}^{2}{ }_{c} / r^{2}$.

$\mathrm{r}_{\mathrm{w}}[\mathrm{m}]=\quad$ Radio efectivo de la rejilla. Si se encuentra cubierta con empaque de grava el radio efectivo sería $\mathrm{r}_{\mathrm{w}}=$ $\left[\left(\mathrm{r}_{\mathrm{p}}^{2}+\mathrm{r}_{\mathrm{e}}^{2}\right) / 2\right]^{1 / 2}$.

$\mathrm{r}_{\mathrm{c} .}[\mathrm{m}]=\quad$ Radio efectivo de almacenamiento $=\left[\left(\mathrm{r}_{\mathrm{p}}^{2}+\mathrm{r}_{\mathrm{a}}^{2}\right) / 2\right]^{1 / 2}$

$\mathrm{r}_{\mathrm{p}}[\mathrm{m}]=\quad$ Radio de perforación.

$\mathrm{r}_{\mathrm{e} .}[\mathrm{m}]=\quad$ Radio de rejilla.

$\mathrm{r}_{\mathrm{a}}[\mathrm{m}]=\quad$ Radio de ademe, donde se encuentra el nivel de agua

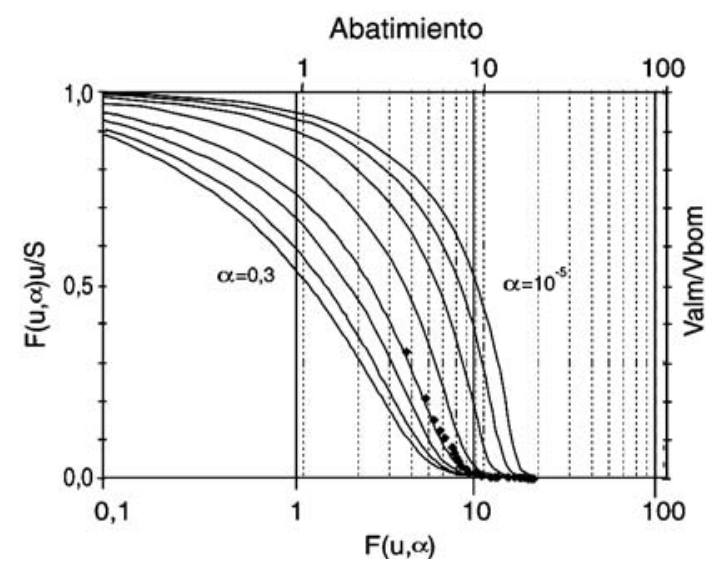

Fig. 3: Familia de curvas para analizar pruebas de bombeo en pozos con almacenamiento de agua en la perforación o ademe. Los valores de a cada una de las curvas, de izquierda a derecha son los siguientes: $\mathrm{a}=0,3 ; \mathrm{a}=0,2 ; \mathrm{a}=0,1 ; \mathrm{a}=0,05$; $\mathrm{a}=0,01 ; \mathrm{a}=10^{-3} ; \mathrm{a}=10^{-4} ; \mathrm{a}=10^{-5}$.

Qt $\left[\mathrm{m}^{3}\right]=\quad$ Volumen bombeado en un tiempo t. Análisis de pruebas de bombeo

Los siguientes son los pasos a seguir para analizar una prueba de bombeo con el método propuesto:

1) Con la ecuación (7) se calculan los valores de $s \pi r^{2}{ }_{w} /(Q t)$ con datos obtenidos en la prueba de bombeo.

2) En un papel semilogarítmico de igual escala y número de ciclos que las curvas patrón, se grafica la curva generada por los 
valores de $s \pi r^{2}{ }_{w} /(Q t)$, en escala aritmética (eje vertical) y los valores de abatimiento (s) en la escala logarítmica (eje horizontal).

3) La curva patrón (Fig 3) se sobrepone a la curva obtenida en el punto anterior, permitiendo únicamente el desplazamiento en el sentido horizontal, de la curva patrón. Los desplazamientos se han de realizar deslizando el eje $\mathrm{F}(\mathrm{u} ; \mathrm{a}) \mathrm{u} / \mathrm{S}=1$ de la curva patrón, sobre el eje Volumen almacenado / Volumen bombeado $=1 \mathrm{de}$ la prueba de bombeo, hasta alcanzar la curva patrón que mejor ajuste a la curva obtenida en el punto (2).

4) Con ambas curvas sobrepuestas se determina el valor de $\alpha$ correspondiente a la curva patrón de mejor ajuste, lo que viene a determinar el coeficiente de almacenamiento con la siguiente ecuación:

$$
S=\frac{\alpha r_{c}^{2}}{r_{w}^{2}}
$$

$r_{w}[m]=$ Radio efectivo de la rejilla (o parte abierta en el acuífero captado) del pozo

$$
r_{w}=\sqrt{\frac{\left(r_{p}^{2}+r_{e}^{2}\right)}{2}}
$$

$\mathrm{r}_{\mathrm{c}}[\mathrm{m}]=$ Radio efectivo del almacenamiento (ademe) en donde se encuentra el nivel de agua. Si el ademe se encuentra cubierto con empaque de grava, el radio efectivo de almacenamiento sería:

$$
r_{c}=\sqrt{\frac{\left(r_{a}^{2}+r_{p}^{2}\right)}{2}}
$$

$\mathrm{r}_{\mathrm{p}}[\mathrm{m}]=$ Radio de perforación .

$\mathrm{r}_{\mathrm{e}}[\mathrm{m}]=$ Radio de rejilla .

$\mathrm{r}_{\mathrm{a}}[\mathrm{m}]=$ Radio de ademe, donde se encuentra el nivel de agua.

Generalmente, el radio de la rejilla es igual al radio del ademe, entonces $S=\alpha$

5) Para obtener el valor de la transmisibilidad (T), con las curvas sobrepuestas, se selecciona un punto cualquiera, determinando el abatimiento (s) y el valor correspondiente de la función $\mathrm{F}(\mathrm{u} ; \mathrm{a})$ de donde:

$$
T=\frac{F(u ; \alpha) Q}{4 \pi s}
$$

$$
\begin{array}{ll}
\mathrm{T}\left[\mathrm{m}^{2} / \text { día }\right]= & \text { Transmisibilidad } \\
\mathrm{s}[\mathrm{m}]= & \begin{array}{l}
\text { Abatimiento del punto seleccionado } \\
\mathrm{F}(\mathrm{u} ; \alpha)=
\end{array} \\
\begin{array}{l}
\text { Función de pozo del punto selec- } \\
\text { cionado }
\end{array} \\
\mathrm{Q}\left[\mathrm{m}^{3} / \mathrm{dí}\right]=\text { Caudal de bombeo }
\end{array}
$$

6) Cuando los valores de $F(u, \alpha)$ son menores de 0,05 , el efecto del almacenamiento prácticamente desaparece y la curva se ajusta a la de Theis. En estos casos, es preferible analizar la prueba por los métodos tradicionales, Theis o Jacob.

\section{Ejemplos:}

Se realiza la siguiente prueba de bombeo (Cuadro 2) a caudal constante $(9,54 \mathrm{l} / \mathrm{s})$ en un pozo perforado en $0,15 \mathrm{~m}$, debidamente engravado, con rejilla y ademe de $0,10 \mathrm{~m}$. El nivel estático se encontraba a $20,18 \mathrm{~m}$.

Cuadro 2

Abatimientos medidos durante la prueba de bombeo y valores calculados de Volumen almacenado / volumen bombeado

\begin{tabular}{ccccccccc}
\hline $\mathrm{t}(\mathrm{min})$ & Abat. (m) & Val/Vbom & $\mathrm{t}(\mathrm{min})$ & Abat. (m) & Val/Vbom & $\mathrm{t}(\mathrm{min})$ & Abat. (m) & Val/Vbom \\
\hline 1 & 3,72 & $3,32 * 10-1$ & 11 & 7,07 & $5,73 \mathrm{E}^{*} 10-2$ & 120 & 9,82 & $7,29 * 10-3$ \\
2 & 4,77 & $2,13 * 10-1$ & 13 & 7,21 & $4,94 \mathrm{E}^{*} 10-2$ & 240 & 10,97 & $4,07 * 10-3$ \\
3 & 5,22 & $1,55 * 10-1$ & 15 & 7,29 & $4,33 * 10-2$ & 300 & 11,38 & $3,38 * 10-3$ \\
4 & 5,72 & $1,27 * 10-1$ & 20 & 7,59 & $3,38^{*} 10-2$ & 360 & 11,72 & $2,90 * 10-3$ \\
5 & 6,02 & $1,07 * 10-1$ & 30 & 8,03 & $2,39 * 10-2$ & 420 & 11,87 & $2,52 * 10-3$ \\
7 & 6,62 & $8,43 * 10-2$ & 40 & 8,35 & $1,86 * 10-2$ & 1320 & 13,79 & $9,31 * 10-3$ \\
9 & 6,82 & $6,75 * 10-2$ & 60 & 8,85 & $1,31 * 10-2$ & 1380 & 13,85 & $8,95 * 10-4$ \\
\hline
\end{tabular}


$\mathrm{r}_{\mathrm{w}}[\mathrm{m}]=$ Radio efectivo de rejilla $=$

$$
\sqrt{\frac{\left(r_{p}^{2}+r_{e}^{2}\right)}{2}}=\sqrt{\frac{\left(0,15^{2}+0,10^{2}\right)}{2}}=0,127 \mathrm{~m}
$$

$r_{c}[\mathrm{~m}]=$ Radio efectivo de almacenamiento $=$

$$
\sqrt{\frac{\left(r_{p}^{2}+r_{a}^{2}\right)}{2}}=\sqrt{\frac{\left(0,15^{2}+0,10^{2}\right)}{2}}=0,127 \mathrm{~m}
$$

$\mathrm{r}_{\mathrm{a}}[\mathrm{m}]=$ Radio del ademe $=0,10$

$\mathrm{r}_{\mathrm{p}}[\mathrm{m}]=$ Radio de perforación $=0,15$

$\mathrm{r}_{\mathrm{e}}[\mathrm{m}]=$ Radio de rejilla $=0,10$

$\mathrm{Q}\left[\mathrm{m}^{3} / \mathrm{min}\right]=$ Caudal bombeado $=$

$$
9,54 * 60 / 1000=0,572
$$

$$
\frac{\text { Volumen almacenado }}{\text { Volumen bombeado }}=\frac{s \pi r_{w}^{2}}{Q t}=\frac{0,051 s}{0,572 t}
$$

Los valores de Volumen almacenado / Volumen bombeado son calculados para cada valor del tiempo de bombeo y tabulados en el cuadro 2. Estos valores se grafican en las ordenadas en escala aritmética, versus los valores de abatimiento (s) en las abscisas en escala logarítmica, luego se sobrepone la curva patrón, como se muestra en la figura 4.

En la figura 4, la curva de mejor ajuste corresponde a un valor de $\alpha=0,05$; por ser el ademe y rejilla del mismo diámetro. El radio medio expuesto a la formación productora $\left(\mathrm{r}_{\mathrm{w}}\right)$ igual al radio efectivo del almacenamiento agua $\left(r_{c}\right)$ tenemos que el coeficiente de almacenamiento es igual a:

$$
S=\alpha\left(\frac{r_{c}}{r_{w}}\right)^{2}=0,05 \cdot\left(\frac{0,127}{0,127}\right)^{2}=0,05
$$

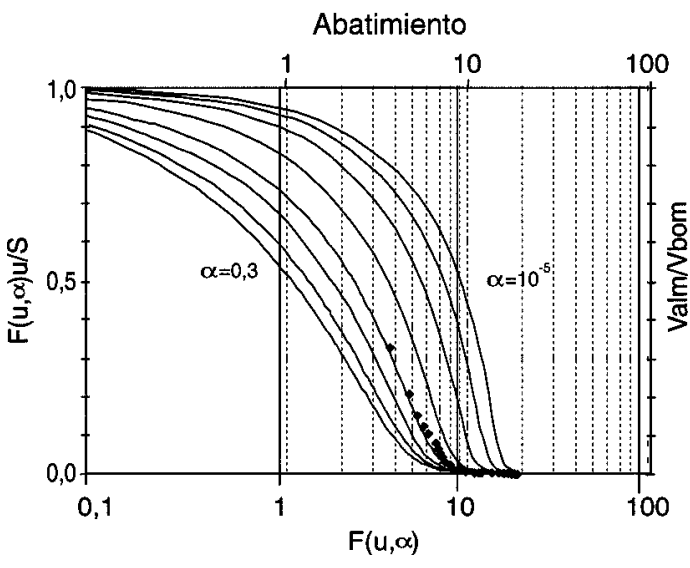

Fig. 4: Sobreposición de los datos de la prueba de bombeo del ejemplo 1, sobre la curva patrón de pozos con almacenamiento de agua en el pozo. Obsérvese que este pozo tiene un radio de ademe y rejilla de $10 \mathrm{~cm}$. El valor del coeficiente de almacenamiento obtenido en el pozo de bombeo es de $\mathrm{S}=$ 0,05 y el de transmisibilidad de $\mathrm{T}=65,63 \mathrm{~m}^{2} /$ día.

Para calcular la transmisibilidad se selecciona un punto en la curva patrón, donde se obtiene el valor de s y de la función $\mathrm{F}(\mathrm{u} ; \alpha)$.

$$
\begin{gathered}
\mathrm{s}=10 \mathrm{~m} \\
\mathrm{~F}(\mathrm{u} ; \alpha)=10 \\
\mathrm{Q}=824,26 \mathrm{~m}^{3} / \mathrm{d} \dot{a} \mathrm{a} \\
T=F(u ; \alpha) \frac{Q}{4 \pi s}=\frac{10 \cdot 824,26}{4 \cdot 3,14 \cdot 10}=65,63 \quad \frac{\mathrm{m}^{2}}{\mathrm{di} a}
\end{gathered}
$$

Se realiza la siguiente prueba de bombeo (Cuadro 3) a caudal constante (1,9 1/s) en un pozo excavado en $0,80 \mathrm{~m}$, con rejilla perforada y ademe de concreto sin empaque de grava con

Cuadro 3

Abatimientos medidos durante la prueba de bombeo y valores calculados (Volumen almacenado / Volumen bombeado)

\begin{tabular}{ccccccccc}
\hline $\mathrm{t}(\mathrm{min})$ & Abat. (m) & Val/Vbom & $\mathrm{t}(\mathrm{min})$ & Abat. (m) & Val/Vbom & $\mathrm{t}(\mathrm{min})$ & Abat. (m) & Val/Vbom \\
\hline 1 & 0,235 & $9,35^{*} 10-1$ & 11 & 1,48 & $5,35^{*} 10-1$ & 50 & 2,355 & $1,87^{*} 10-1$ \\
2 & 0,425 & $8,45^{*} 10-1$ & 13 & 1,61 & $4,93 * 10-1$ & 60 & 2,41 & $1,60^{*} 10-1$ \\
3 & 0,595 & $7,89 * 10-1$ & 15 & 1,725 & $4,57 * 10-1$ & 90 & 2,5 & $1,10^{*} 10-1$ \\
4 & 0,745 & $7,41 * 10-1$ & 20 & 1,935 & $3,85 * 10-1$ & 120 & 2,56 & $8,48^{*} 10-1$ \\
5 & 0,885 & $7,04 * 10-1$ & 25 & 2,06 & $3,28^{*} 10-1$ & 200 & 2,665 & $5,30^{*} 10-2$ \\
7 & 1,125 & $6,39 * 10-1$ & 30 & 2,16 & $2,86^{*} 10-1$ & 240 & 2,695 & $4,47 * 10-2$ \\
9 & 1,32 & $5,83^{*} 10-1$ & 40 & 2,28 & $2,27 * 10-1$ & 290 & 2,745 & $3,76^{*} 10-2$ \\
\hline
\end{tabular}


0,76 m de diámetro interior. El nivel estático se encontraba a $17,12 \mathrm{~m}$.

Al no tener empaque de grava y ser un pozo excavado, en que el almacenamiento y la rejilla son del mismo diámetro, los radios efectivos de la rejilla y del ademe son iguales al radio interno del tubo de concreto con que se ademó el pozo.

$$
\begin{array}{ll}
\mathrm{r}_{\mathrm{w}}[\mathrm{m}]= & \begin{array}{l}
\text { Radio efectivo de rejilla }=0,38 \mathrm{~m} \\
\mathrm{r}_{\mathrm{c}}[\mathrm{m}]=
\end{array} \\
\begin{array}{l}
\text { Radio efectivo de almacenamiento } \\
=0,38 \mathrm{~m}
\end{array} \\
\begin{array}{ll}
\mathrm{r}_{\mathrm{p}}[\mathrm{m}]= & \begin{array}{l}
\text { Radio de perforación }=0,40 \mathrm{~m} \\
\mathrm{r}_{\mathrm{e}}[\mathrm{m}]=
\end{array} \\
\mathrm{Q}\left[\mathrm{m}^{3} / \mathrm{min}\right]= & \text { Radio de rejilla }=0,38 \mathrm{~m} \\
=0,114 \mathrm{~m}^{3} / \mathrm{min}
\end{array} \\
\frac{\text { Volumen almacenado }}{\text { Volumen bombeado }}=\frac{s \pi r_{w}^{2}}{Q t}=\frac{0,453}{0,114 t}
\end{array}
$$

Los valores de Volumen almacenado/Volumen bombeado son calculados para cada valor del tiempo de bombeo y tabulados en el cuadro 3. Estos valores se grafican en las ordenadas en escala aritmética, versus los valores de abatimiento (s) en las abscisas en escala logarítmica, luego se sobrepone la curva patrón, como se muestra en la figura 5 .

De la figura 5, la curva de mejor ajuste corresponde a un valor de $\alpha=0,0001$; por ser el

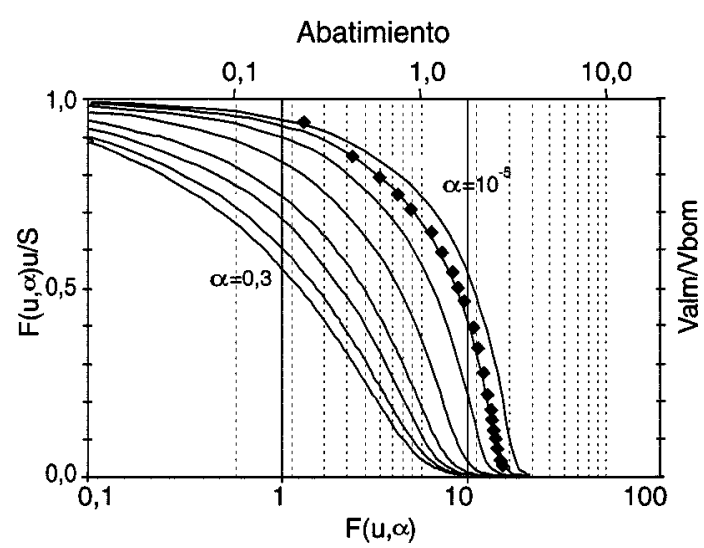

Fig. 5: Sobreposición de los datos de la prueba de bombeo del ejemplo 2, sobre la curva patrón de pozos con almacenamiento de agua en el pozo. El pozo es excavado y tiene un radio de $38 \mathrm{~cm}$ de ademe y rejilla. El valor del coeficiente de almacenamiento obtenido en el pozo de bombeo es de $\mathrm{S}=$ 0,0001 y el de transmisibilidad de $\mathrm{T}=67,37 \mathrm{~m}^{2} /$ día. ademe y rejilla del mismo diámetro. El radio medio expuesto a la formación productora $\left(\mathrm{r}_{\mathrm{w}}\right)$ es igual al radio efectivo del almacenamiento agua $\left(r_{c}\right)$ tenemos que el coeficiente de almacenamiento es igual a:

$$
\begin{gathered}
S=\alpha\left(\frac{r_{c}}{r_{w}}\right)^{2}=0,0001\left(\frac{0,38}{0,38}\right)^{2}=0,0001 \\
\mathrm{r}_{\mathrm{c}}[\mathrm{m}]=\text { Radio efectivo de almacenamiento }= \\
0,38 \mathrm{~m}
\end{gathered}
$$

Para calcular la trasmisibilidad se selecciona un punto en la curva patrón, donde se obtiene el valor de s y de la función $\mathrm{F}(\mathrm{u} ; \alpha)$.

$$
\begin{gathered}
\mathrm{s}=1,94 \mathrm{~m} \\
\mathrm{~F}(\mathrm{u} ; \alpha)=10 \\
\mathrm{Q}=164,16 \mathrm{~m}^{3} / \mathrm{día} \\
T=F(u ; \alpha) \frac{Q}{4 \pi s}=10 \cdot \frac{164,16}{4 \cdot 3,14 \cdot 1,94}=67,39 \frac{\mathrm{m}^{2}}{\text { día }}
\end{gathered}
$$

\section{CONCLUSIONES}

El método propuesto utiliza una nueva función Volumen almacenado / Volumen bombeado (ecuación 3), que permite determinar con mayor precisión el coeficiente de almacenamiento en pozos de gran diámetro.

Cuando el valor de Volumen almacenado / Volumen bombeado es menor de 0,05 , el efecto de almacenamiento prácticamente ha desaparecido, por lo que la prueba de bombeo puede ser analizada por los métodos convencionales de Theis o Jacob.

El método permite analizar pruebas de bombeo de corta duración, en pozos de gran diámetro, sin problemas de que la curva se sobreponga a la misma vez en diferentes curvas patrón.

Con valores de Volumen almacenado / Volumen bombeado mayores de 0,05 este método permite determinar el coeficiente de almacenamiento en los pozos de bombeo, aún de diámetros de perforación generalmente utilizados $(15-25 \mathrm{~cm})$ que con los métodos convencionales no permiten la determinación del coeficiente de almacenamiento en forma precisa para estos diámetros. 


\section{REFERENCIAS}

ALLAN FREEZE, A. \& CHERRY, J.A., 1979: Groundwater. - 604 págs. Prentice Hall, New Jersey.
KRUSEMAN, G.P. \& DE RIDDER, N.A.,1994: Analysis and evaluation of pumping test data. - 377 págs. Inter. Inst. Land Reclamation and Improvement, The Netherlands. 